\title{
Pseudo-Anosov extensions and degree one maps between hyperbolic surface bundles
}

\author{
BY MICHEL BOILEAU \\ Laboratoire Émile Picard, \\ Université Paul Sabatier, TOULOUSE Cedex 4, France \\ e-mail: boileau@picard.ups-tlse.fr \\ YI NI \\ Department of Mathematics, \\ Princeton University, NJ 08544, U. S. A. \\ e-mail: yni@math.princeton.edu \\ AND SHICHENG WANG \\ LMAM, Department of Mathematics, \\ Peking University, Beijing 100871 China \\ e-mail: wangsc@math.pku.edu.cn
}

\begin{abstract}
Let $F^{\prime}, F$ be any two closed orientable surfaces of genus $g^{\prime}>g \geq 1$, and $f: F \rightarrow F$ be any pseudo-Anosov map. Then we can "extend" $f$ to be a pseudo-Anosov map $f^{\prime}: F^{\prime} \rightarrow$ $F^{\prime}$ so that there is a fiber preserving degree one map $M\left(F^{\prime}, f^{\prime}\right) \rightarrow M(F, f)$ between the hyperbolic surface bundles. Moreover the extension $f^{\prime}$ can be chosen so that the surface bundles $M\left(F^{\prime}, f^{\prime}\right)$ and $M(F, f)$ have the same first Betti numbers.
\end{abstract}

\section{Introduction}

All surfaces are oriented and all automorphisms on surfaces are orientation preserving.

Let $F$ be an oriented closed surface of genus $g \geq 1$, and $f: F \rightarrow F$ be an automorphism. We denote the surface bundle with fiber $F$ and monodromy $f$ by $M(F, f)$.

Definition 1.1. Suppose $G$ is a compact surface of genus $g \geq 1$. A circle $c$ on $G$ is essential if $c$ is neither contractible nor boundary parallel. An automorphism $f$ of $G$ is pseudo-Anosov if $f^{n}(c)$ is not isotopic to $c$ for any essential circle $c \subset G$ and any integer $n$. (Note in the case $G$ is a torus, the term "pseudo-Anosov" we define here is usually known as "Anosov".)

Remark 1·2. Our definition of pseudo-Anosov maps is slightly different from the more standard definition in the literature. Pseudo-Anosov maps in our sense should be considered as "maps isotopic to a pseudo-Anosov map" in the standard sense.

Profound theories of Nielsen-Thurston and of Thurston in 2- and 3-dimensional topology tell us that pseudo-Anosov is the most important class of surface automorphisms, and when $\chi(F)<0, M(F, f)$ is a hyperbolic 3-manifold if and only if $f$ is pseudo-Anosov. 
THEOREM 1.3. Let $F_{s}, F_{t}$ be closed orientable surfaces of genus $g_{s}, g_{t}$ respectively, $g_{s}>g_{t} \geq 1$, and $f_{t}: F_{t} \rightarrow F_{t}$ be a pseudo-Anosov map. Then

(1) There exists a hyperbolic 3-manifold $M\left(F_{s}, f_{s}\right)$, such that the is a fiber preserving degree one map $P: M\left(F_{s}, f_{s}\right) \rightarrow M\left(F_{t}, f_{t}\right)$. (Here the subscript s means "source", and the subscript $t$ means "target".)

(2) Moreover the $f_{s}$ in (1) can be chosen so that $M\left(F_{s}, f_{s}\right)$ and $M\left(F_{t}, f_{t}\right)$ have the same first Betti numbers.

Motivation for Theorem 1.3 is from [1, where the following facts were proved:

(1) For each 3-manifold $M$, there is a degree one map $f: M\left(F_{s}, f_{s}\right) \rightarrow M$ such that $M\left(F_{s}, f_{s}\right)$ is hyperbolic and $\beta_{1}\left(M\left(F_{s}, f_{s}\right)\right)=\beta_{1}(M)+1$.

(2) If there is a degree one map $f: M\left(F_{s}, f_{s}\right) \rightarrow M$ with $\beta_{1}\left(M\left(F_{s}, f_{s}\right)\right)=\beta_{1}(M)$ and $M$ is irreducible, then $M$ is a surface bundle and $f$ can be homotoped to a fiber preserving one.

It is natural to wonder how to find fiber preserving degree one maps between nonhomeomorphic hyperbolic surface bundles (of the same first Betti numbers). In Section 2, we will prove Theorem 1.3 (1). In Section 3, by modifying the proof in Section 2, we will prove Theorem $1.3(2)$.

The proof of Theorem 1.3 relies on an extension process from the pseudo-Anosov map $f_{t}$ on $F_{t}$ to a pseudo-Anosov map $f_{s}$ on $F_{s}$, which is delicate and somewhat complicated.

We will outline this process, i.e., for given $M\left(F_{t}, f_{t}\right)$ and $F_{s}$, how to find $f_{s}$. In this outline we assume that $g_{t} \geq 2$. This process in Section 2 is divided into three steps.

Step 1. Fix a disk $D \subset F_{t}$ and let $V=F_{t}-\operatorname{int}(D)$. We can assume that $f_{t}|D=\operatorname{id}| D$ up to isotopy. Then as a restriction of a pseudo-Anosov map, $f_{t} \mid V: V \rightarrow V$ is a pseudoAnosov map (Lemma 2·4).

Step 2. We will construct two embedding $e_{0}, e_{1}: V \rightarrow F_{s}$ such that $(1) e_{0}(\partial V)$ and $e_{1}(\partial V)$ are not homotopic in $F_{s},(2)$ two pinches $p_{0}, p_{1}: F_{s} \rightarrow F_{t}$ (see Definition 2.1) defined by $p_{j} \circ e_{j}=\mathrm{id}_{V}: V \rightarrow V$ are homotopic (Lemma 2.6).

Step 3. The two embeddings $e_{1}$ and $e_{2}$ in step 2 also provided a homeomorphism $\bar{f}_{t}:=e_{1} \circ f_{t} \mid \circ e_{0}^{-1}: e_{0}(V) \rightarrow e_{1}(V)$. With properties of $e_{1}$ and $e_{2}$ described in Step 2, we will be able to extend $\bar{f}_{t}$ to a pseudo-Anosov map $f_{s}: F_{s} \rightarrow F_{s}$ (Proposition [2.7).

Then clearly $p_{1} \circ f_{s}=f_{t} \circ p_{0}$, hence there exists a fiber preserving degree one map $P: M\left(F_{s}, f_{s}\right) \rightarrow M\left(F_{t}, f_{t}\right)$ (Lemma 2.3). This finishes the proof of Theorem 1.3 (1).

Now we give more detailed outline of the extension process in Step 3, on which the proof of Theorem $1 \cdot 3$ (2) is based.

Let $\bar{f}_{s}: F_{s} \rightarrow F_{s}$ be any extension of $\bar{f}_{t}: e_{0}(V) \rightarrow e_{1}(V)$ (Lemma 2.4). Let $W_{1}=$ $F_{s}-\operatorname{int}_{1}(V)$ and $h: W_{1} \rightarrow W_{1}$ be any pseudo-Anosov map. Let $\mathcal{A}_{1}$ be any maximal independent set of disjoint circles on $W_{1}$ (see Definition [2.9), let $\tau\left(\mathcal{A}_{1}\right)$ be a composition of Dehn twists along all components in $\mathcal{A}_{1}$. Then $f_{s}=\tau^{l}\left(\mathcal{A}_{1}\right) \circ h^{k} \circ \bar{f}_{s}$ is pseudo Anosov

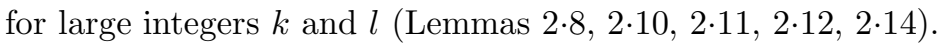

In Section 3, we choose $\bar{f}_{s}, h$ and $\mathcal{A}_{1}$ carefullly so that Theorem 1.3 (2) is proved (Lemmas $3 \cdot 1,3 \cdot 2$ 3.3).

We end the introduction by a comment on a related work $[\mathbf{9}$. The main result in $\mathbf{9}$ is that for an orientable closed surface $F$ with $\chi(F)<0$ and two non-isotopic circles $c$ and $c^{\prime}$ on $F$, if $g(c)=c^{\prime}$ for some automorphism $g$ on $F$, then $f(c)=c^{\prime}$ for some pseudoAnosov map $f$ on $F$. Some arguments in proving Lemmas $2 \cdot 10$ and $2 \cdot 12$ were influenced 
by that in $[\mathbf{9}$. Indeed $[\mathbf{9}$ is produced in a rather earlier stage of understanding the present project.

Acknowledgements. We are grateful to Dr. Hao Zheng for drawing the figures in this paper. M.Boileau and S.C.Wang wish to thank Prof. D.Gabai and Prof. J.Mess for helpful conversations.

Y.Ni is partially supported by a Centennial fellowship of the Graduate School at Princeton University. S.C.Wang is partially supported by MSTC.

Y.Ni joined this project when he was a graduate student at Peking University. The paper was finished when Y.Ni visited Peking University.

\section{Homotopic pinches and pseudo-Anosov extensions}

Definition $2 \cdot 1$. Let $D$ be a fixed disc in $F_{t}$ and $V=F_{t}-\operatorname{int}(D)$. A degree one map $p: F_{s} \rightarrow F_{t}$ is a pinch if $p \mid: p^{-1}(V) \rightarrow V$ is a homeomorphism.

It has been known since Nielsen and Kneser that every degree one map between surfaces is homotopic to a pinch, see [2] for a reference.

Notation 2.2. In the rest of this paper, $r=s, t$ and $j=0,1$.

Recall that $M\left(F_{r}, f_{r}\right)=F_{r} \times[0,1] / f_{r}^{\prime}$, where $f_{r}^{\prime}: F_{r} \times\{0\} \rightarrow F_{r} \times\{1\}$ is given by $f_{r}^{\prime}(x, 0)=\left(f_{r}(x), 1\right)$. Let $q_{r}: F_{r} \times[0,1] \rightarrow F_{r}$ be the projection defined by $q_{r}(x, u)=x$, and $e_{r, j}: F_{r} \rightarrow F_{r} \times\{j\} \subset F_{r} \times[0,1]$ be the homeomorphism given by $e_{r, j}(x)=(x, j)$. Let $o_{r}: F_{r} \times[0,1] \rightarrow M\left(F_{r}, f_{r}\right)$ be the quotient map and $F_{r}^{\prime}=o_{r}\left(F_{r} \times 0\right)=o_{r}\left(F_{r} \times 1\right)$.

Then

$$
q_{r} \circ f_{r}^{\prime} \circ e_{r, 0}=f_{r}, \quad e_{r, j} \circ q_{r}=\operatorname{id}_{F_{r} \times\{j\}} .
$$

Lemma 2.3. There exists a fiber preserving degree one map $P: M\left(F_{s}, f_{s}\right) \rightarrow M\left(F_{t}, f_{t}\right)$ if and only if there are homotopic pinches $p_{0}, p_{1}: F_{s} \rightarrow F_{t}$ such that $p_{1} \circ f_{s}=f_{t} \circ p_{0}$.

Proof. Suppose first that $P: M\left(F_{s}, f_{s}\right) \rightarrow M\left(F_{t}, f_{t}\right)$ is a fiber preserving degree 1 map. Up to homotopy we may assume that $P^{-1}\left(F_{t}^{\prime}\right)=F_{s}^{\prime}$ and $P \mid: F_{s}^{\prime} \rightarrow F_{t}^{\prime}$ is a pinch. Moreover we may assume that the induced degree one map on $S^{1}$ is orientation preserving. Then by cutting $M\left(F_{r}, f_{r}\right)$ along $F_{r}^{\prime}, P$ provides a proper degree one map

$$
\bar{P}:\left(F_{s} \times[0,1], F_{s} \times\{0\}, F_{s} \times\{1\}\right) \rightarrow\left(F_{t} \times[0,1], F_{t} \times\{0\}, F_{t} \times\{1\}\right)
$$

with the property $\left.\bar{P}\right|_{F_{s} \times\{1\}} \circ f_{s}^{\prime}=\left.f_{t}^{\prime} \circ \bar{P}\right|_{F_{s} \times\{0\}}$.

Let $p_{j}=\left.q_{t} \circ \bar{P}\right|_{F_{s} \times\{j\}} \circ e_{s, j}: F_{s} \rightarrow F_{t}$. Then $p_{j}$ is a pinch and $q_{t} \circ \bar{P}: F_{s} \times[0,1] \rightarrow F_{t}$ is a homotopy from $p_{0}$ to $p_{1}$. Moreover $\left.\bar{P}\right|_{F_{s} \times\{1\}} \circ f_{s}^{\prime}=\left.f_{t}^{\prime} \circ \bar{P}\right|_{F_{s} \times\{0\}}$ and (*) imply that

$$
\begin{aligned}
p_{1} \circ f_{s} & =\left.q_{t} \circ P\right|_{F_{s} \times\{1\}} \circ e_{s, 1} \circ q_{s} \circ f_{s}^{\prime} \circ e_{s, 0} \\
& =\left.q_{t} \circ \bar{P}\right|_{F_{s} \times\{1\}} \circ f_{s}^{\prime} \circ e_{s, 0} \\
& =\left.q_{t} \circ f_{t}^{\prime} \circ \bar{P}\right|_{F_{s} \times\{0\}} \circ e_{s, 0} \\
& =\left.q_{t} \circ f_{t}^{\prime} \circ e_{t, 0} \circ q_{t} \circ \bar{P}\right|_{F_{s} \times\{0\}} \circ e_{s, 0} \\
& =f_{t} \circ p_{0} .
\end{aligned}
$$

Suppose then there are two homotopic pinches $p_{0}, p_{1}: F_{s} \rightarrow F_{t}$ such that $p_{1} \circ f_{s}=$ $f_{t} \circ p_{0}$. Let $P^{\prime}: F_{s} \times[0,1] \rightarrow F_{t}$ be a homotopy from $p_{0}$ to $p_{1}$. Then $P^{\prime}$ provides a proper degree one map

$$
\bar{P}:\left(F_{s} \times[0,1], F_{s} \times\{0\}, F_{s} \times\{1\}\right) \rightarrow\left(F_{t} \times[0,1], F_{t} \times\{0\}, F_{t} \times\{1\}\right)
$$


defined by $\bar{P}(x, u)=\left(P^{\prime}(x, u), u\right)$. Clearly $\bar{P}$ is fiber preserving and $p_{j}=\left.q_{t} \circ \bar{P}\right|_{F_{s} \times\{j\}} \circ$ $e_{s, j}$. Then $p_{1} \circ f_{s}=f_{t} \circ p_{0}$ and $(*)$ implies that

$$
\left.q_{t} \circ P\right|_{F_{s} \times\{1\}} \circ e_{s, 1} \circ q_{s} \circ f_{s}^{\prime} \circ e_{s, 0}=\left.q_{t} \circ f_{t}^{\prime} \circ e_{t, 0} \circ q_{t} \circ \bar{P}\right|_{F_{s} \times\{0\}} \circ e_{s, 0},
$$

hence

$$
\left.q_{t} \circ P\right|_{F_{s} \times\{1\}} \circ f_{s}^{\prime} \circ e_{s, 0}=\left.q_{t} \circ f_{t}^{\prime} \circ \bar{P}\right|_{F_{s} \times\{0\}} \circ e_{s, 0} .
$$

Since $\left.q_{t}\right|_{F_{t} \times\{1\}}$ and $e_{s, 0}$ are invertible, we have

$$
\left.P\right|_{F_{s} \times\{1\}} \circ f_{s}^{\prime}=\left.f_{t}^{\prime} \circ \bar{P}\right|_{F_{s} \times\{0\}} .
$$

Hence $\bar{P}$ is able to induce a fiber preserving degree one map $P: M\left(F_{s}, f_{s}\right) \rightarrow M\left(F_{t}, f_{t}\right)$.

By Lemma 2.3 to prove Theorem 1.3 (1), we need only to find two homotopic pinches $p_{0}, p_{1}: F_{s} \rightarrow F_{t}$ and a pseudo-Anosov map $f_{s}: F_{s} \rightarrow F_{s}$ such that $p_{1} \circ f_{s}=f_{t} \circ p_{0}$.

For $D \subset F_{t}$ and $V=F_{t}-\operatorname{int}(D)$ given in Definition 2.1] we can assume that $f_{t} \mid D=$ id up to isotopy.

Lemma 2.4. If $f_{t}: F_{t} \rightarrow F_{t}$ is a pseudo-Anosov map, then $f_{t} \mid V: V \rightarrow V$ is also a pseudo-Anosov map.

Proof. Suppose there is a non-contractible circle $c$ on $V$ such that $\left.f_{t}\right|^{n}(c) \sim c$ on $V$ for some $n>0$, then $f_{t}^{n}(c) \sim c$ on $F_{t}$. Since $f_{t}$ is pseudo-Anosov, $c$ is contractible on $F_{t}$. Hence $c$ bounds a disc $D^{*}$ in $F_{t}$ and $D \subset D^{*}$. It follows that $c=\partial D^{*}$ is parallel to $\partial D=\partial V$. Hence $f_{t} \mid: V \rightarrow V$ is pseudo-Anosov by definition.

Let $p_{0}, p_{1}: F_{s} \rightarrow F_{t}$ be two pinches. Then the pull-back of $V$ into $F_{s}$ provides embeddings $e_{j}: V \hookrightarrow F_{s}$. Let $V_{j}=e_{j}(V), W_{j}=F_{s}-\operatorname{int}\left(V_{j}\right),(j=0,1)$. The following lemma is clear

LEMMA $2 \cdot 5$.

$$
\bar{f}_{t}:=e_{1} \circ f_{t} \circ e_{0}^{-1}: V_{0} \rightarrow V_{1}
$$

is a homeomorphism. Moreover, $\bar{f}_{t}$ can be extended to a homeomorphism $f_{s}: F_{s} \rightarrow F_{s}$, such that $f_{t} \circ p_{0}=p_{1} \circ f_{s}$.

A necessary condition to guarantee the extension $f_{s}$ in Lemma 2.5 to be pseudo-Anosov is that $e_{0}(\partial D)$ is not homotopic to $e_{1}(\partial D)$.

Now with Lemma $2 \cdot 3$ and Lemma 2.5] Theorem [1.3 (1) follows from the following Lemma 2.6 and Proposition 2.7

Lemma 2.6. With the notation above, there exist two pinches $p_{0}, p_{1}: F_{s} \rightarrow F_{t}$ such that

(i) $p_{0}$ and $p_{1}$ are homotopic;

(ii) $e_{0}(\partial D)$ is not homotopic to $e_{1}(\partial D)$.

Proof. We will find two essential circles $\gamma_{0}, \gamma_{1} \subset F_{s}$ such that

(1) $\gamma_{0}$ is not homotopic to $\gamma_{1}$,

(2) $\gamma_{j}$ separates $F_{s}$ into 1-punctured surfaces $V_{j}$ and $W_{j}$, where $V_{0}, V_{1}$ have genus $g_{t}$.

Then we define the pinch $p_{j}: F_{s} \rightarrow F_{t}$ such that $W_{j}$ is the pinched part.

Case 1. $g_{t} \geq 2 . W_{j}, V_{j}$, are shown in Figure 1 .

Let $p_{j}: F_{s} \rightarrow F_{t}$ be a pinch which sends $W_{j}$ to $D_{j} \subset F_{t}$ such that the restrictions 
$p_{0}\left|, p_{1}\right|: F_{s} \backslash\left(W_{0} \cup W_{1}\right) \rightarrow F_{t} \backslash\left(D_{0} \cup D_{1}\right)$ are identical homeomorphisms. This requirement can be reached if we consider $p_{j}: F_{s} \rightarrow F_{t}$ as a quotient map which is the identity on $F_{s} \backslash W_{j}$ and pinches $W_{j}$ to $D_{j}$.

Note $W_{0} \cup W_{1}$ is a compact surface with two boundary components and $D_{0} \cup D_{1}$ is annulus. Moreover the restrictions $p_{0}\left|, p_{1}\right|: W_{0} \cup W_{1} \rightarrow D_{0} \cup D_{1}$ are degree one maps which are identity on the boundary, it follows from classical argument that $p_{0}\left|, p_{1}\right|$ : $W_{0} \cup W_{1} \rightarrow D_{0} \cup D_{1}$ are homotopic relative to the boundary, and finally $p_{0}, p_{1}: F_{s} \rightarrow F_{t}$ are homotopic.

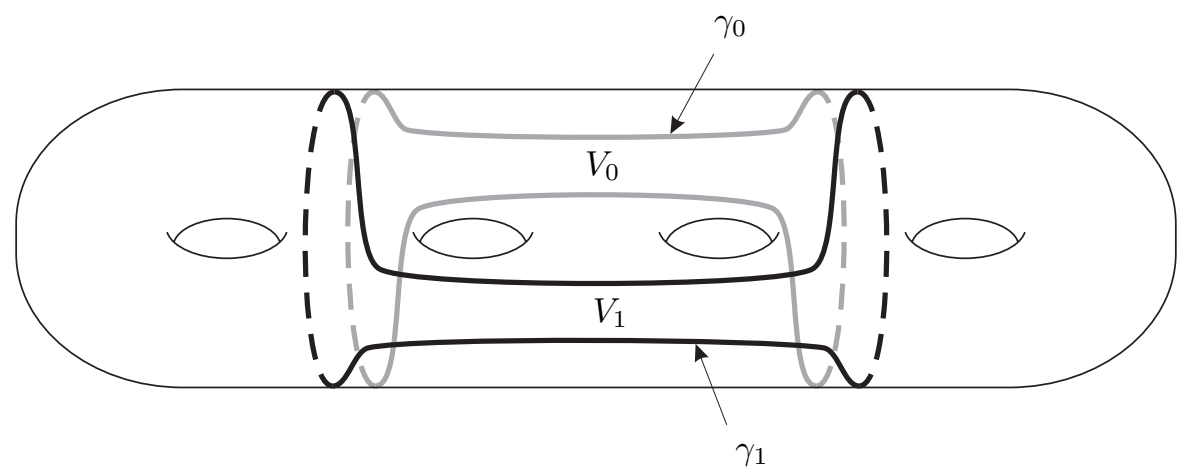

Figure 1

Case 2. $g_{t}=1$. Then $\pi_{1}\left(F_{t}\right)=\mathrm{H}_{1}\left(F_{t}\right)$ is abelian and for each map $p: F_{s} \rightarrow F_{t}$, $p_{\pi}: \pi_{1}\left(F_{s}\right) \rightarrow \pi_{1}\left(F_{t}\right)$ is a composition of $\sigma: \pi_{1}\left(F_{s}\right) \rightarrow \mathrm{H}_{1}\left(F_{s}\right)$ and $p_{\#}: \mathrm{H}_{1}\left(F_{s}\right) \rightarrow \mathrm{H}_{1}\left(F_{t}\right)$, where $\sigma$ is the abelianizing map, $p_{\#}$ is the map on homology. So the homotopy class of $p$ is determined by $p_{\#}$ by elementary homotopy theory (see [3]).

Using this fact, we can construct $\gamma_{0}$ and $\gamma_{1}$ as following: choose essential curves $\alpha, \beta_{0}$ and $\beta_{1}$ on $F_{s}$, see Figure 2 , such that

(1) $\beta_{0}$ and $\beta_{1}$ are in the same homology class, but not in the same homotopy class;

(2) $\left|\alpha \cap \beta_{0}\right|=\left|\alpha \cap \beta_{1}\right|=1$.

Let $\gamma_{j}=\partial N\left(\alpha \cup \beta_{j}\right), V_{j}=N\left(\alpha \cup \beta_{j}\right)$. It is easy to check that $\gamma_{0} \nsim \gamma_{1}$ and $p_{0 \#}=p_{1 \#}$ : $\mathrm{H}_{1}\left(F_{s}\right) \rightarrow \mathrm{H}_{1}\left(F_{t}\right)$.

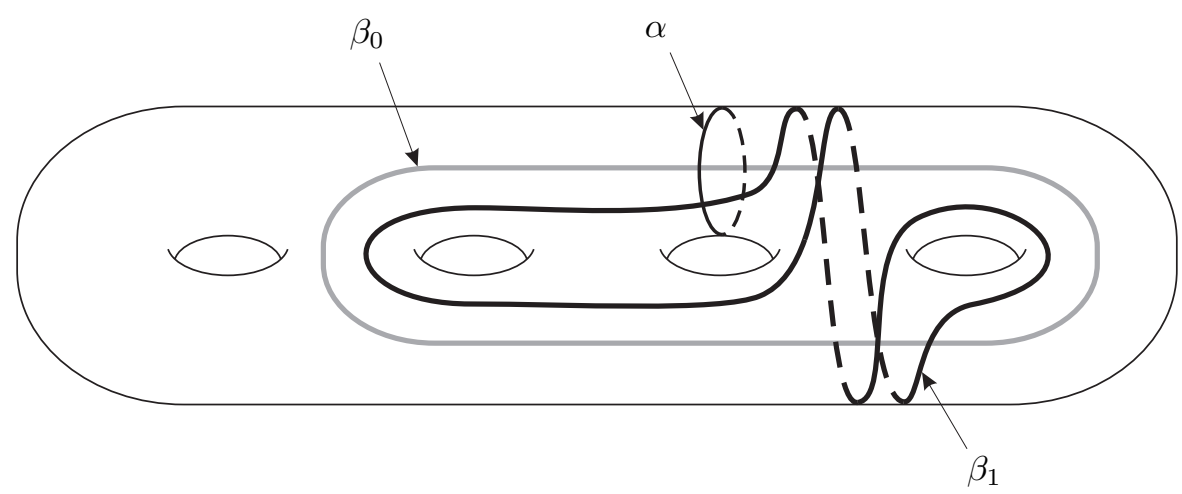

Figure 2

Proposition 2.7. With the notation as above, once the two pinches $p_{0}, p_{1}: F_{s} \rightarrow F_{t}$ 
are chosen to meet (i) and (ii) in Lemma [2.6. the extension $f_{s}$ in Lemma [2.5 can be chosen to be pseudo-Anosov.

Suppose the two pinches $p_{0}, p_{1}: F_{s} \rightarrow F_{t}$ are chosen to meet (i) and (ii) in Lemma 2.6] and $f_{s}:\left(F_{s}, V_{0}\right) \rightarrow\left(F_{s}, V_{1}\right)$ is an extension of $\bar{f}_{t}=e_{1} \circ f_{t} \mid \circ e_{0}^{-1}: V_{0} \rightarrow V_{1}$ with the condition $p_{1} \circ f_{s}=f_{t} \circ p_{0}$.

Lemma 2.8. (1) If $c$ is an essential circle in $V$, then $c$ is essential in $F_{t}$.

(2) No non-trivial circle $c \subset V_{j}$ can be isotoped into $W_{j^{\prime}}, j, j^{\prime} \in\{0,1\}, j \neq j^{\prime}$.

Proof. (1) Otherwise $c$ would bound a disk $D^{*}$ in $F_{t}$ with $\partial V \subset D^{*}$, hence $c$ is parallel to $\partial V$ in $V$, a contradiction.

(2) Otherwise say $c \subset V_{0}$ is a non-trivial circle, which is isotopic to a circle $c^{\prime} \subset W_{1}$ in $F_{s}$.

First suppose that $c$ is essential in $V_{0}$. By $p_{0} \sim p_{1}$ we have $p_{0}(c) \sim p_{1}\left(c^{\prime}\right)$. On one hand $c$ is essential in $V_{0}$ implies that $p_{0}(c)$ is essential in $V_{0}$, and then $p_{0}(c)$ is essential in $F_{t}$ by (1). But on the other hand, $c^{\prime} \subset W_{1}$ implies that $p_{1}\left(c^{\prime}\right)$ is homotopically trivial. We reach a contradiction.

Then suppose that $\partial W_{1}$ can be isotoped into $W_{0}$. Then one of the two components $V_{1}$ and $W_{1}$ must be contained in $W_{0}$. Since $W_{0}$ and $W_{1}$ are homeomorphic, if $W_{1} \subset W_{0}$, we must have $\partial W_{0}$ is parallel to $\partial W_{1}$, a contradiction. Hence $V_{1} \subset W_{0}$, which implies that $\pi_{1}\left(V_{1}\right) \subset \operatorname{ker} p_{0 \pi}=\operatorname{ker} p_{1 \pi}$, which clearly is impossible.

So what remains to us is to modify $\left.f_{s}\right|_{W_{0}}$.

Definition 2.9. 9] A set of mutually disjoint circles $\mathcal{C}=\left\{c_{1}, \ldots, c_{m}\right\}$ on a compact surface $F$ is an independent set, if the circles in $\mathcal{C}$ are essential and mutually non-parallel.

Lemma 2.10. Let $h: W_{1} \rightarrow W_{1}$ be a pseudo-Anosov map which is the identity in $\partial W_{1}$. We extend $h$ by identity to an automorphism $h$ of $F_{s} . \mathcal{A}$ is a maximal independent set of circles in $W_{0} . f=f_{s}: F_{s} \rightarrow F_{s}$ is an extension of $\bar{f}_{t}: V_{0} \rightarrow V_{1}$.

Then when $k$ is sufficiently large, for any $\alpha \in \mathcal{A}, h^{k} f(\alpha)$ is not isotopic to any circle in $\mathcal{A}$.

Proof. Suppose $k_{1}<k_{2}, \alpha \in \mathcal{A}$. We claim that $h^{k_{1}} f(\alpha) \nsim h^{k_{2}} f(\alpha)$ in $F_{s}$. In fact, $h^{k_{1}} f(\alpha)$ is an essential curve in $W_{1}$, and any two curves in $W_{1}$, which are homotopic in $F_{s}$, must be homotopic in $W_{1}$. But $\left.h\right|_{W_{1}}$ is a pseudo-Anosov automorphism on $W_{1}$, so $h^{k_{2}-k_{1}}\left(h^{k_{1}} f(\alpha)\right)$ is not isotopic to $h^{k_{1}} f(\alpha)$.

Hence for any $\alpha \in \mathcal{A}$, there are only finitely many $k$, such that $h^{k} f(\alpha)$ is homotopic to a circle in $\mathcal{A}$. Hence the conclusion holds.

From now on we replace $f$ by $h^{k} f$.

Let $\mathcal{A}_{0}$ be a maximal independent set of circles on $W_{0}, \mathcal{A}_{1}$ be its image under $f_{s}$. Let $V_{j}^{\prime}=e_{s, j}\left(V_{j}\right), W_{j}^{\prime}=e_{s, j}\left(W_{j}\right), \mathcal{A}_{j}^{\prime}=e_{s, j}\left(\mathcal{A}_{j}\right)$. Let $L=o_{s}\left(\mathcal{A}^{\prime} \bigcup \partial W_{0}^{\prime}\right), V^{\prime}=o_{s}\left(V_{j}^{\prime}\right)$, $W^{\prime}=o_{s}\left(W_{j}^{\prime}\right), F^{\prime}=o_{s}\left(F_{s} \times\{j\}\right) ;$ and $X=M\left(F_{s}, f_{s}\right)-\operatorname{int}(N(L)), F^{*}=F^{\prime} \cap X$. Then

$$
f_{s}^{\prime}:\left(F_{s} \times\{0\}, V_{0}^{\prime}, W_{0}^{\prime}, \mathcal{A}_{0}\right) \rightarrow\left(F_{s} \times\{1\}, V_{1}^{\prime}, W_{1}^{\prime}, \mathcal{A}_{1}\right)
$$

is a homeomorphism. We will prove that $X$ is hyperbolic. We first have

Lemma 2.11. (1) $\mathcal{A}_{j}^{\prime}$ is a maximal independent set of circles on $W_{j}^{\prime}$ and each nontrivial circle in $F^{*}$ is either essential in $V_{j}^{\prime}$ or parallel to a component of $\partial W_{j}^{\prime} \cup \mathcal{A}_{j}^{\prime}$.

(2) No component of $\partial W_{0} \cup \mathcal{A}_{0}$ is homotopic to a component of $\partial W_{1} \cup \mathcal{A}_{1}$, in $F_{s}$. 
Proof. (1) follows directly from the definitions and the constructions.

(2) $\partial W_{1}$ (resp. $\left.\partial W_{0}\right)$ can not be isotoped into $W_{0}$ (resp. $W_{1}$ ) by Lemma 2.8 (2). No component of $\mathcal{A}_{1}=f_{s}\left(\mathcal{A}_{0}\right)$ is isotopic to a component of $\mathcal{A}_{0}$ by Lemma 2.10 Hence $(2)$ follows.

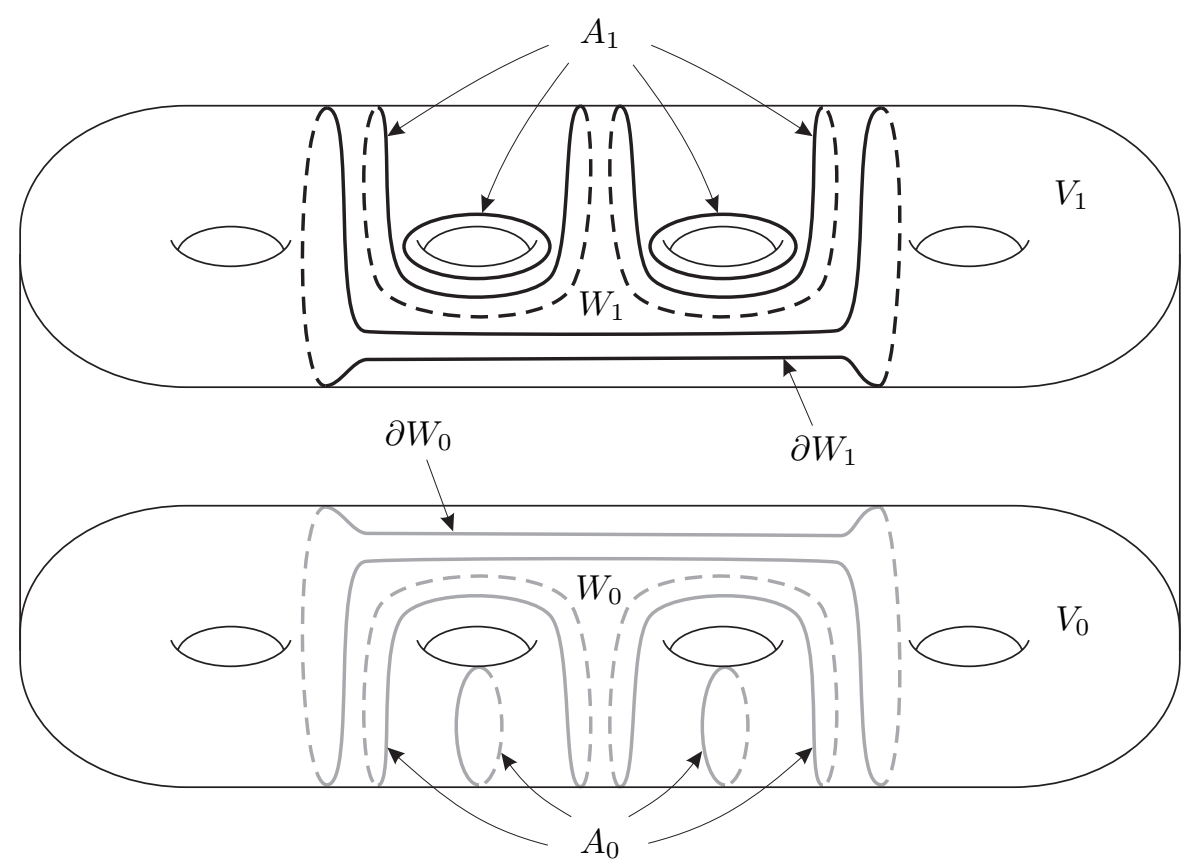

Figure 3

Figure 3 is what happens in $F_{s} \times[0,1]$, and clearly illustrate the meaning of Lemma $2 \cdot 11$

Lemma $2 \cdot 12$. $X$ is atoroidal.

Proof. Suppose that $T$ is an essential torus in $X$. We assume that $T$ has been isotoped in $X$ so that $\left|T \cap F^{*}\right|$ is minimal. Then $T \cap F^{*}=\mathcal{C}^{*}$ consists of $\pi_{1}$-injective circles on both $T$ and $F^{*}$. Note $\mathcal{C}^{*} \neq \emptyset$, otherwise $T$ would be an incompressible torus in $F_{s} \times[0,1]$, which is impossible.

Cutting $X$ along $F^{*}$, we get a manifold $X^{\prime} \subset F_{s} \times[0,1]$ and $T \backslash \mathcal{C}$ is a collection of annuli $A_{1}, A_{2}, \ldots, A_{n} \subset X^{\prime}$. In the case $A_{k}$ is vertical, we denote the component of $\partial A_{k}$ in $F_{s} \times\{j\}$ by $c_{k, j}$, and $q_{s}\left(c_{k, 0}\right)=q_{s}\left(c_{k, 1}\right) \subset F_{s}$ by $c_{k}$.

Now we claim that if one component of $\partial A_{k}$ is essential in $V_{j}^{\prime}$, then $A_{k}$ is vertical. Because otherwise $\partial A_{k} \subset V_{j}^{\prime}$, and we can push $A_{k}$ across $V^{\prime}$ in $X$ to reduce $\left|T \cap F^{*}\right|$, a contradiction.

By Lemma 2.11 (1), there are two cases:

Case 1. Some component of $\partial A_{k}$ is an essential circle on $V_{j}^{\prime}$.

By the claim above, $A_{k}$ is vertical. Then $f_{s}^{\prime}\left(c_{k, 0}\right)$ is a component of $\partial A_{l}$. $(l=k-1$ or $k+1$.) Since $f_{s}^{\prime}\left(c_{k, 0}\right)$ is essential in $V_{1}^{\prime}$, it follows by the claim $A_{l}$ is vertical and $f_{s}^{\prime}\left(c_{k, 0}\right)=c_{l, 1}$. Then $f_{s}\left(c_{k}\right)=c_{l}$ and $c_{l}$ is essential in $V_{1}$. Hence $c_{l}$ can not be isotoped into $W_{0}$ by Lemma $2 \cdot 8$ (2). Since $c_{l, 0}$ is disjoint from $\partial W_{0}^{\prime}, c_{l} \subset V_{0}$. Clearly $c_{l}$ is still an 
essential circle in $V_{0}$, and therefore $c_{l, 0}$ is essential in $V_{0}^{\prime}$. Since $T$ is connected, repeat the same argument finitely many times, we get that

(1) all $A_{k}$ are vertical;

(2) all $c_{k, 0}$ are essential in $V_{0}^{\prime}$ and all $c_{k, 1}$ are essential in $V_{1}^{\prime}$;

(3) $f_{s}^{\prime}\left(c_{k, 0}\right)=c_{k+1,1}$ (re-indexing $A_{k}$ if needed, and the subscript $k$ is considered mod $n$ ). Hence each $c_{k}$ is essential in both $V_{0}$ and $V_{1}$, and $f_{s}\left(c_{k}\right)=c_{k+1}$.

Now both $p_{0}\left(c_{k}\right)$ and $p_{1}\left(c_{k}\right)$ are essential circles in $V$, and therefore essential in $F_{t}$ by Lemma 2.8 (1). Since $f_{t} \circ p_{0}=p_{1} \circ f_{s}$, we have that $f_{t} \circ p_{0}\left(c_{k}\right)=p_{1} \circ f_{s}\left(c_{k}\right)=p_{1}\left(c_{k+1}\right)$. Since $p_{0}$ and $p_{1}$ are homotopic, we have $p_{0}\left(c_{k}\right) \sim p_{1}\left(c_{k}\right)$. Then up to isotopy $f_{t}^{n} \circ p_{0}\left(c_{k}\right)=$ $p_{0}\left(c_{k}\right)$, which contradicts to the fact that $f_{t}$ is a pseudo-Anosov map on $F_{t}$.

Case 2. Each component of $\partial A_{k}$ is parallel to a component of $\partial W_{0}^{\prime} \cup \mathcal{A}_{0}^{\prime}$. By Lemma 2.11 (2), no $A_{k}$ is vertical, hence both components of $\partial A_{k}$ are parallel to a component $c$ of $\partial W_{0}^{\prime} \cup \mathcal{A}_{0}^{\prime}$. So $A_{k}$ can be rel $\partial A_{k}$ isotoped into $N(c)$ in $X^{\prime}$. Hence back to $X$ the torus $T$ can be isotoped into $N\left(o_{s}(c)\right)$. This means that $T$ is boundary parallel in $X$, contrary to our assumption.

It is easy to see that $X$ is irreducible: a reducing sphere $S$ would bound a ball $B$ in $M\left(F_{s}, f_{s}\right)$, because $M\left(F_{s}, f_{s}\right)$, as a surface bundle over circle, is irreducible. Hence $B$ would contain some component of $L$. This is impossible because each component of $L$ is essential in $M\left(F_{s}, f_{s}\right)$.

$X$ is not a Seifert fibered space: it contains $q\left(F_{s} \times \frac{1}{2}\right)$, a non-separating, hyperbolic, closed incompressible surface. No such surface exists in a Seifert fibered space with boundary, because an essential surface in such a manifold is either horizontal (hence bounded) or vertical (hence a torus or an annulus).

Now the geometrization theorem of Thurston for Haken manifolds [8] leads us to the following:

Corollary $2 \cdot 13 . X$ is a hyperbolic manifold.

Suppose $L=\left\{\alpha_{1}, \ldots, \alpha_{m}\right\}$, let $T_{l}$ be the torus $\partial N\left(\alpha_{l}\right)$ on $\partial X, l=1, \ldots, m$. Denote by $\tau_{c}$ the right hand Dehn twist along a circle $c$ on $F_{s}$. Pick a meridian-longitude pair for each $T_{l}$, with longitude a component of $F^{\prime} \cap T_{l}$. $q_{l}$ is a slope on $T_{l}$, define $X\left(q_{1}, \ldots, q_{m}\right)$ to be the manifold obtained by $q_{l}$ Dehn filling on $T_{l}$. The following lemma points a well-known relation between Dehn fillings and Dehn twists, which has been used in some papers, say [4] and $[$ ].

Lemma $2 \cdot 14$. Let $\tilde{f}\left(k_{1}, \ldots, k_{m}\right)=\tau_{\alpha_{1}}^{k_{1}} \circ \cdots \circ \tau_{\alpha_{m}}^{k_{m}} \circ f$. Then

$$
M\left(F_{s}, \tilde{f}\left(k_{1}, \ldots, k_{n}\right)\right)=X\left(1 / k_{1}, \ldots, 1 / k_{m}\right)
$$

for all $k_{l} \in \mathbb{Z}$.

Proof of Proposition 2.7. By Corollary [2.13] $X$ is a hyperbolic manifold, therefore, by the hyperbolic surgery theorem of Thurston $\mathbf{7}$, $X\left(1 / k_{1}, \ldots, 1 / k_{m}\right)$ is hyperbolic for sufficiently large $k_{l}$. The previous lemma implies that

$$
X\left(1 / k_{1}, \ldots, 1 / k_{m}\right)=M\left(F_{s}, \tilde{f}\left(k_{1}, \ldots, k_{m}\right)\right) .
$$

The theorem now follows from Thurston's theorem that $M\left(F_{s}, \tilde{f}\right)$ is hyperbolic if and only if $\tilde{f}$ is isotopic to a pseudo-Anosov map $(\underline{\mathbf{8}}, \mathbf{5})$. 


\section{Adjusting Betti numbers}

Now we pay attention to the Betti numbers of the surface bundles. Using HHN extension one can calculate directly that

$$
\mathrm{H}_{1}(M(F, f) ; \mathbb{Z})=\mathrm{H}_{1}(F, \mathbb{Z}) / \operatorname{ker}\left(I_{2 g}-f_{\#}\right) \oplus \mathbb{Z}
$$

where $g=g(F), I_{2 g}$ is the unit matrix in $S L_{2 g}(\mathbb{Z})$.

By abuse of notation, denote the image of $\mathrm{H}_{1}\left(V_{j}\right)$ in $\mathrm{H}_{1}\left(F_{s}\right)$ by $\mathrm{H}_{1}\left(\widehat{V}_{j}\right)$. Similarly, define $\mathrm{H}_{1}\left(\widehat{W}_{j}\right)$. We have

$$
\mathrm{H}_{1}\left(F_{s}\right)=\mathrm{H}_{1}\left(\widehat{V}_{0}\right) \oplus \mathrm{H}_{1}\left(\widehat{W}_{0}\right)=\mathrm{H}_{1}\left(\widehat{V}_{1}\right) \oplus \mathrm{H}_{1}\left(\widehat{W}_{1}\right) .
$$

LEMMA $3 \cdot 1$.

$$
\begin{aligned}
\mathrm{H}_{1}\left(\widehat{V}_{0}\right) & =\mathrm{H}_{1}\left(\widehat{V}_{1}\right), \\
\mathrm{H}_{1}\left(\widehat{W}_{0}\right) & =\mathrm{H}_{1}\left(\widehat{W}_{1}\right),
\end{aligned}
$$

as subgroups of $\mathrm{H}_{1}\left(F_{s}\right)$.

Proof. The second equation is easy, because $\mathrm{H}_{1}\left(\widehat{W}_{j}\right)=\operatorname{ker} p_{j \#}$, and $p_{0} \sim p_{1}$ implies $p_{0 \#}=p_{1 \#}$.

Now we will prove the first equation. Suppose $c_{0} \subset V_{0}$ is a circle, then $c=p_{0}\left(c_{0}\right)$ is a circle in $V$, thus $c_{1}=p_{1}^{-1}(c)$ is a circle in $V_{1}$. Since $p_{0} \sim p_{1}$, we have a map $H: F_{s} \times[0,1] \rightarrow F_{t}, H(\cdot, j)=p_{j}(\cdot)$.

Make $H$ transverse to $c$, then $H^{-1}(c)$ is a submanifold of $F_{s} \times[0,1]$ with boundary $c_{0} \cup c_{1}$. So $c_{0}$ and $c_{1}$ represent the same homology class in $\mathrm{H}_{1}\left(F_{s} \times[0,1]\right)=\mathrm{H}_{1}\left(F_{s}\right)$. Hence the generators of $\mathrm{H}_{1}\left(\widehat{V}_{0}\right)$ is the same as the ones of $\mathrm{H}_{1}\left(\widehat{V}_{1}\right)$, our conclusion holds.

Choose a basis of $\mathrm{H}_{1}\left(\widehat{V}_{0}\right)$ and a basis of $\mathrm{H}_{1}\left(\widehat{W}_{0}\right)$ to make up a basis of $\mathrm{H}_{1}\left(F_{s}\right)$. Under this basis, $f_{s \#}$ will be represented by a matrix of the form:

$$
\left(\begin{array}{cc}
f_{t \#} & 0 \\
0 & A
\end{array}\right) \text {. }
$$

Lemma $3 \cdot 2$. The map $f_{s}$ can be chosen so that the matrix $I-A$ is non-degenerate.

Proof. Let $\delta=g_{s}-g_{t}$. Choose curves $\alpha_{1}, \ldots, \alpha_{\delta}, \beta_{1}, \ldots, \beta_{\delta} \subset W_{1}$, such that they are mutually disjoint, except that $\alpha_{l}$ intersects $\beta_{l}$ in a single point transversely. These $2 \delta$ curves form a symplectic basis of $\mathrm{H}_{1}\left(\widehat{W}_{1}\right)$. Under this basis, the intersection form of $\mathrm{H}_{1}\left(\widehat{W}_{1}\right)$ is $\left(\begin{array}{cc}0 & I_{\delta} \\ -I_{\delta} & 0\end{array}\right)$. So if $f:\left(F_{s}, W_{0}\right) \rightarrow\left(F_{s}, W_{1}\right)$ is a homeomorphism, $\left.f_{\#}\right|_{\mathrm{H}_{1}\left(\widehat{W}_{0}\right)}$ will be represented by a symplectic matrix $F$.

We choose a map $\eta: W_{1} \rightarrow W_{1}$, such that it fixes the points on $\partial W_{1}$, and it induces $F^{-1}$ on homology.

When $\delta>1$, by Theorem 2 in $\underline{\mathbf{6}}$, every symplectic matrix of rank $2 \delta$ can be represented by a pseudo-Anosov map on a closed surface of genus $\delta$. So there is a pseudo-Anosov map $h: W_{1} \rightarrow W_{1}$, such that it fixes the points on $\partial W_{1}$, and induces $-I_{2 \delta}$ on homology. Extend $\eta, h$ to maps on $F_{s}$, with the points in $V_{1}$ fixed.

Let $\gamma_{l}=\partial N\left(\alpha_{l} \cup \beta_{l}\right)$, which is a separating circle in $W_{1}$ (see those separating circles in $\mathcal{A}_{1}$, Figure 3 ). Now we extend $\left\{\alpha_{1}, \ldots, \alpha_{\delta}, \gamma_{1}, \ldots, \gamma_{\delta}\right\}$ to a maximal independent set $\mathcal{A}$ on $W_{0}$. Then every curve in $\mathcal{A}-\left\{\alpha_{1}, \ldots, \alpha_{\delta}\right\}$ is homologous to 0 . Let $L=\mathcal{A} \cup\left\{\partial W_{1}\right\}$ So the only Dehn twists along circles in $L$, which act nontrivially on homology group, 
are $\tau_{\alpha_{1}}, \ldots, \tau_{\alpha_{\delta}}$. The action of products of these twists on $\mathrm{H}_{1}\left(\widehat{W}_{1}\right)$ is represented by a upper-triangular matrix $T$, whose diagonal elements are all 1. By Lemma 2.10 $h^{2 k+1} \eta f$ does not send any curve in $L$ into $L$ when $k$ is sufficiently large. The matrix of $h^{2 k+1} \eta f$, when restricted on $\mathrm{H}_{1}\left(\widehat{W}_{0}\right)$, is $-I_{2 \delta}$. Now replace $f$ by the composition of Dehn twists along $L$ and $h^{2 k+1} \eta f$, we have

$$
I_{2 \delta}-A=I_{2 \delta}+T
$$

is non-degenerate.

When $\delta=1$, we give a direct construction. Let $\alpha, \beta$ be a symplectic basis of $W_{0}$.

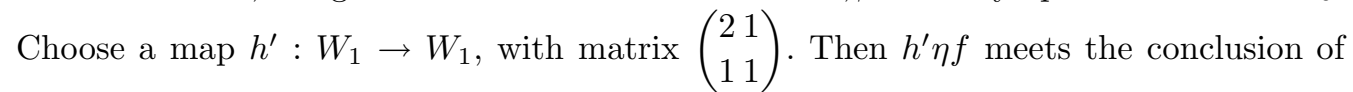
Lemma 2.10 $\tau$ is the Dehn twist along $\eta f(\alpha)$, then the matrix of $\tau$ is $\left(\begin{array}{ll}1 & 1 \\ 0 & 1\end{array}\right) \cdot \tau_{0}$ is the Dehn twist along $\partial W_{1}$. Now the matrix $A$ of $\tau_{0}^{m} \tau^{k} h^{\prime} \eta f$ is

$$
\left(\begin{array}{ll}
1 & 1 \\
0 & 1
\end{array}\right)^{k}\left(\begin{array}{ll}
2 & 1 \\
1 & 1
\end{array}\right)=\left(\begin{array}{cc}
2+k & 1+k \\
1 & 1
\end{array}\right) .
$$

One can check $I-A$ is non-degenerate when $k \geq 0$.

To prove Theorem $1 \cdot 3$ (2), we need only to show the following lemma.

Lemma 3.3. With the notation as above, the extension $f_{s}$ in Proposition 2.7 can be chosen so that

$$
\operatorname{rank}\left(\mathrm{H}_{1}\left(F_{s} ; \mathbb{Q}\right) / \operatorname{ker}\left(I_{2 g_{s}}-f_{s \#}\right)\right)=\operatorname{rank}\left(\mathrm{H}_{1}\left(F_{t}, \mathbb{Q}\right) / \operatorname{ker}\left(I_{2 g_{t}}-f_{t \#}\right)\right) .
$$

Proof. The conclusion follows from the formula of computing $\mathrm{H}_{1}(M(F, f))$ and Lemma $3 \cdot 2$

Proof of Theorem [1.3. This theorem follows from Proposition 2.7 and Lemma 3.3

\section{REFERENCES}

[1] M. Boileau and S.C. Wang. Non-zero degree maps and surface bundles over $S^{1}$. J. Diff. Geom. 43 (1996) 789-806.

[2] A. Edmonds. Deformation of maps to branched coverings in dimension two. Ann. of Math. 110 (1979) 113-124.

[3] S.-T. Hu. Homotopy theory. (Academic Press, 1959).

[4] D. D. Long and H. R. MorTon. Hyperbolic 3-manifolds and surface automorphisms. Topology. 25 (1986) no. 4 575-583.

[5] J. P. Otal. Le theoreme d'hyperbolisation pour les variétés fibrées de dimension 3. Astérisque 235 Soc. Mat. de France (1997).

[6] A. Papadopoulos. Difféomorphismes pseudo-Anosov et automorphismes symplectiques de l'homologie. (French) Ann. Sci. École Norm. Sup. $4^{e}$ Sér. 15 no.3 (1982) 543-546.

[7] W. Thurston. The geometry and topology of 3-manifolds. (Princeton University, 1978).

[8] W. Thurston. Three-dimentional manifolds, Kleinian groups and hyperbolic geometry. Bull. Amer. Math. Soc. 6 (1982) 357-381.

[9] S. Wang, Y.-Q. Wu and Q. Zhou. Pseudo-Anosov maps and simple curves on surfaces. Math. Proc. Camb. Phil. Soc. 128 (2000) 321-326. 